\title{
Stabilité de l'holonomie sans structure de Frobenius : cas des courbes
}

\author{
Daniel CARO
}

\begin{abstract}
By using Christol and Mebkhout's algebrization and finiteness theorem, we prove that in the case of smooth curves, Berthelot's strongest conjecture on the stability of holonomicity is still valid without Frobenius structure but under some non-Liouville type hypotheses.
\end{abstract}

\section{Introduction}

Soit $\mathcal{V}$ un anneau de valuation discrète complet d'inégales caractéristiques $(0, p)$, de corps résiduel $k$ supposé parfait, de corps des fractions $K$. Soient $\mathfrak{X}$ un $\mathcal{V}$-schéma formel lisse, $X_{0}$ sa fibre spéciale, $T_{0}$ un diviseur de $\mathfrak{X}$, Ul l'ouvert de $\mathfrak{X}$ complémentaire de $Z_{0}$. Berthelot a construit le faisceau sur $\mathfrak{X}$ des opérateurs différentiels de niveau fini et d'ordre infini noté $\mathcal{D}_{\mathfrak{X}, Q}^{\dagger}$; ce dernier correspondant à la tensorisation par $Q$ (indiqué par l'indice $Q$ ) du complété faible $p$-adique (indiqué par le symbole «†») du faisceau classique $\mathcal{D}_{\mathfrak{X}}$ des opérateurs différentiels sur $\mathfrak{X}$. Enfin, en ajoutant des singularités surconvergentes le long de $Z_{0}$, il construit le faisceau $\mathcal{D}_{\mathfrak{X}}^{\dagger}\left({ }^{\dagger} Z_{0}\right)_{\mathrm{Q}}$ sur $\mathfrak{X}$ (voir [Ber96b] ou [Ber02]). On désigne par $F-D_{\text {coh }}^{\mathrm{b}}\left(\mathcal{D}_{\mathfrak{X}}^{\dagger}\left({ }^{\dagger} Z_{0}\right)_{Q}\right)$ la catégorie des complexes de $\mathcal{D}_{\mathfrak{X}}^{\dagger}\left({ }^{\dagger} Z_{0}\right)_{\mathrm{Q}}$-modules (toujours à gauche par défaut) à cohomologie cohérente et bornée munie d'une structure de Frobenius, i.e. d'un isomorphisme $\mathcal{D}_{\mathcal{X}}^{\dagger}\left({ }^{\dagger} Z_{0}\right)_{\mathrm{Q}}$-linéaire de la forme $F^{*}(\mathcal{E}) \stackrel{\sim}{\longrightarrow} \mathcal{E}$ avec $F^{*}$ désignant l'image inverse par l'endomorphisme (ou une puissance) du Frobenius absolu $X_{0} \rightarrow X_{0}$.

Soit $\mathcal{E} \in F-D_{\text {coh }}^{\mathrm{b}}\left(\mathcal{D}_{\mathcal{X}}^{\dagger}\left({ }^{\dagger} Z_{0}\right)_{\mathrm{Q}}\right)$. Berthelot a conjecturé (voir [Ber02, 5.3.6.D)]) que le $F$-complexe $\mathcal{E}$ est holonome si et seulement si $\mathcal{E} \mid \mathfrak{U}$ est

(*) Indirizzo dell'A.: Laboratoire de Mathématiques Nicolas Oresme, Université de Caen Campus 2, 14032 Caen Cedex, France.

Email: daniel.caro@unicaen.fr 
holonome. Cette conjecture a été validée dans le cas où $X_{0}$ est une courbe propre (voir [Car06b]) puis une courbe (voir [CT08]) et enfin une variété projective (voir [Car11c]).

Nous nous intéressons ici à une extension sans structure de Frobenius de cette conjecture. D'une part, en reprenant la caractérisation homologique de l'holonomie de Virrion (voir [Vir00]), il est possible d'étendre de manière naturelle la notion d'holonomie en nous affranchissant de la structure de Frobenius. Cependant, si on ne fait aucune hypothèse supplémentaire qui permettent d'exclure les problèmes liés aux nombres de Liouville, la conjecture de Berthelot sans structure de Frobenius est fausse. En effet, lorsque $X_{0}$ est propre, le problème est qu'il existe des $\mathcal{D}_{\mathfrak{X}}^{\dagger}\left({ }^{\dagger} Z_{0}\right)_{\mathbb{Q}}$-modules cohérents, $\mathcal{O}_{\mathfrak{X}}\left({ }^{\dagger} Z_{0}\right)_{\mathbb{Q}}$-cohérents dont les espaces de cohomologie $p$-adique ne sont pas de dimension finie et qui ne sont par conséquent même pas $\mathcal{D}_{\mathfrak{X}, \mathrm{Q}}^{\dagger}$-cohérents (voir l'exemple donné dans 4.7). Mais, nous disposons de théorèmes d'algébrisation et de finitude de Christol-Mebkhout pour les isocristaux surconvergents sur les courbes lisses qui satisfont à certaines hypothèses de non Liouvillité (voir l'introduction de [CM01]). Nous résumons ces hypothèses de non Liouvillité via l'appellation «propriété $(N L-N L)$ ». Par exemple, cette propriété $(N L-N L)$ est satisfaite lorsque l'on dispose d'une structure de Frobenius.

Dans ce papier, nous établissons que la conjecture de Berthelot reste valable sans structure de Frobenius dans le cas des courbes pour les complexes se dévissant en isocristaux surconvergents satisfaisant à la propriété $(N L-N L)$. Précisons à présent son contenu.

Soient $X$ un $\mathcal{V}$-schéma projectif et lisse de dimension relative $1, U$ un ouvert affine et $Z$ le diviseur réduit complémentaire de $U$ dans $X$. On note $\mathfrak{X}$ le $\mathcal{V}$-schéma formel projectif et lisse déduit de $X$ par complétion $p$-adique, on note $X_{0}, U_{0}$ et $Z_{0}$ les fibres spéciales respectives de $X, U$ et $Z$. Ce papier se compose d'une première partie où nous donnons quelques rappels sur les $\mathcal{V}$-schémas formels faibles de Meredith. Par exemple, par complétion faible $p$-adique de $U$, on obtient le $\mathcal{V}$-schéma formel faible affine $U^{\dagger}$.

Dans le deuxième chapitre, nous construisons une équivalence canonique notée $\mathrm{sp}_{+}$de catégories entre d'une part les modules (tensorisés par Q) à connexion sur $U^{\dagger}$ solubles au sens de Christol-Mebkhout et d'autre part les $\mathcal{D}_{\mathfrak{X}}^{\dagger}\left({ }^{\dagger} Z_{0}\right)_{Q}$-modules cohérents qui sont $\mathcal{O}_{\mathfrak{X}}\left({ }^{\dagger} Z_{0}\right)_{\mathrm{Q}}$-modules cohérents (cette dernière catégorie est équivalente à celle des isocristaux surconvergents sur $U_{0}$ ). Remarquons que ces premiers vivent sur le $\mathcal{V}$ schéma formel faible $U^{\dagger}$ tandis que ces seconds vivent sur le $\mathcal{V}$-schéma formel $\mathfrak{X}$. Pour établir une telle équivalence entre ces deux catégories de 
faisceaux, l'ingrédient technique est d'utiliser les théorèmes de type $A$ sur les $\mathcal{V}$-schémas formels faibles (du à Meredith) et sur les $\mathcal{V}$-schémas formels (du à Berthelot dans le cas affine et à Noot-Huyghe dans le cas projectif avec un diviseur ample).

Christol et Mebkhout ont utilisé un foncteur, que l'on appelle ici «foncteur de daguification», qui associe à un module (tensorisé par Q) à connexion sur une variété algébrique affine et lisse sur $\mathcal{V}$ un module à connexion (tensorisé par Q) sur le $\mathcal{V}$-schéma formel faible affine et lisse déduit par complétion faible $p$-adique. Nous étudions dans la troisième partie les propriétés de l'image essentielle de ce foncteur. Le résultat central de cette partie (cela découle en fait des deux lemmes 3.9 et 3.10) est qu'un objet de cette image essentielle est presque holonome : nous prouvons que si $\mathcal{E}^{\dagger}$ est un module (tensorisé par Q) à connexion sur $U^{\dagger}$ qui est de plus dans l'image essentielle de ce foncteur de daguification, alors le $\mathcal{D}_{X}^{\dagger}\left({ }^{\dagger} Z_{0}\right)_{Q}$-module cohérent $\operatorname{sp}_{+}\left(\mathcal{E}^{\dagger}\right)$ correspondant (le foncteur $\mathrm{sp}_{+}$est celui construit dans le deuxième chapitre de ce travail) est à isomorphisme près de la forme $\mathcal{D}_{\mathfrak{X}}^{\dagger}\left({ }^{\dagger} Z_{0}\right)_{\mathrm{Q}} \otimes_{\mathcal{D}_{\mathfrak{X}, Q}^{\dagger}} \mathcal{G}$, avec $\mathcal{G}$ un $\mathcal{D}_{\mathfrak{X}, \mathrm{Q}}^{\dagger}$-module holonome.

Dans une quatrième partie, nous énonçons d'abord le théorème d'algébrisation et le théorème de finitude cohomologique de Christol et Mebkhout. Ce premier théorème signifie que, quitte à faire une extension finie de la base $\mathcal{V}$, les isocristaux surconvergents sur $U_{0}$ (ou plutôt les modules solubles selon leur terminologie) qui satisfont certaines hypothèses de non Liouvillité légèrement plus faibles que la condition $(N L-N L)$ de ci-dessus sont dans l'image essentielle du foncteur de daguification. Leur théorème de finitude se traduit par le fait que la cohomologie $\mathrm{du} \mathcal{D}_{\mathfrak{X}}^{\dagger}\left({ }^{\dagger} Z_{0}\right)_{\mathrm{Q}}$-module cohérent associé à un module soluble via l'équivalence $\mathrm{sp}_{+}$du second chapitre est de dimension finie sur $K$.

Enfin, nous démontrons dans une dernière partie la conjecture de Berthelot sous les conditions $(N L-N L)$. L'idée est de se ramener par dévissage au cas d'un isocristal surconvergent satisfaisant aux conditions $(N L-N L)$. Cela résulte alors (par dévissage cette fois-ci du module holonome $\mathcal{G}$ ci-dessus construit dans la troisième partie : voir la preuve de 5.1) du théorème d'algébrisation de Christol et Mebkhout qui nous permet d'utiliser le troisième chapitre et du théorème de finitude cohomologique de Christol et Mebkhout. En particulier, nous obtenons une nouvelle preuve (on remplace ici le théorème de la réduction semistable de Kedlaya par ceux cités ci-dessus de Christol et Mebkhout) de la conjecture de Berthelot avec structure de Frobenius dans le cas des courbes. 


\section{Notations}

Soit $\mathcal{V}$ un anneau de valuation discrète complet d'inégales caractéristiques $(0, p)$, de corps résiduel $k$ supposé parfait, d'uniformisante $\pi$, de corps des fractions $K$. On note $\mathcal{R}_{K}$ la $K$-algèbre des séries de Laurent $\sum_{k \in Z} a_{k} x^{k}$ à coefficients dans $K$ qui convergent pour tout $x \in K$ tel que $|x| \in[r-\varepsilon, r[$ pour $\varepsilon>0$ non précisé.

Soient $X$ un $\mathcal{V}$-schéma projectif et lisse, $Z$ un diviseur ample de $X$ et $U$ l'ouvert de $X$ complémentaire de $Z$. On suppose $U=\operatorname{Spec} A$ affine et on note $j: U \subset X$ l'inclusion canonique. On désigne par $X^{\dagger}$ et $U^{\dagger}$ les $\mathcal{V}$ schémas formels faibles lisses déduits par complétion faible $p$-adique de respectivement $X$ et $U$ (voir [Mer72] ou 1) ; par respectivement $\mathfrak{X}$ et $\mathfrak{U}$ les complétés $p$-adiques ; par $X_{0}, U_{0}$ et $Z_{0}$ les fibres spéciales respectives de $X$, $U$ et $Z$. On suppose pour simplifier que $X_{0}$ est de dimension pure notée $d_{X_{0}}$. On dispose du morphisme structural $f: \mathfrak{X} \rightarrow \operatorname{Spf} \mathcal{V}$. Les morphismes d'espaces annelés de la forme $X^{\dagger} \rightarrow X$ ou $U^{\dagger} \rightarrow U$ seront notés $\varepsilon$ (voir 1.6.1). On note $j_{0}: U^{\dagger} \subset X^{\dagger}$ l'inclusion canonique. Sans nuire à la généralité, nous supposerons que les $k$-schémas sont toujours réduits.

Nous utiliserons les notations usuelles sur les $\mathcal{D}$-modules arithmétiques que nous ne rappelons pas ici (e.g., voir [Ber02] et le premier chapitre de [Car09]). Nous ne rappelons pas non plus les propriétés bien connues concernant les les $\mathcal{D}$-modules arithmétiques (e.g. voir le premier chapitre de [Car09]). Le module des sections globales de faisceaux de la forme $\mathcal{O}_{*}^{\text {? ou }}$

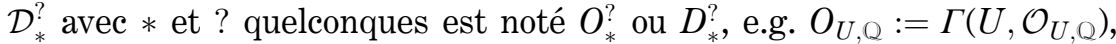
$D_{U, \mathrm{Q}}:=\Gamma\left(U, \mathcal{D}_{U, \mathrm{Q}}\right), D_{U^{\dagger, \mathrm{Q}}}^{\dagger}:=\Gamma\left(U^{\dagger}, \mathcal{D}_{U^{\dagger}, \mathrm{Q}}^{\dagger}\right)$, etc. De plus, pour tout faisceau en groupes $\mathcal{E}$ (resp. pour tout $\mathcal{V}$-module $E$ ), on pose $\mathcal{E}_{\mathrm{Q}}:=\mathcal{E} \otimes_{\mathrm{Z}} \mathrm{Q}$ (resp. $E_{K}:=E \otimes \mathcal{V} K$ ).

\section{Rappels sur les $\mathcal{V}$-schémas formels faibles}

DÉfinition 1.1 (Complétion faible). Si $B$ est une $\mathcal{V}$-algèbre, on note $B^{\dagger}$ le complété faible $p$-adique de $B$ (voir [MW68]). Par exemple, $\mathcal{V}\left[t_{1}, \ldots, t_{d}\right]^{\dagger}$ est l'ensemble des séries entières $f=\sum_{0 \leq i_{1}, \ldots, i_{d}} a_{i_{1} \ldots i_{d}} t_{1}^{i_{1}} \cdots t_{d}^{i_{d}}$ à coefficients dans $\mathcal{V}$ telles que, pour une certaine constante $c$, pour tout $d$-uplet d'entiers $i_{1}, \ldots, i_{d}$ on ait $c v_{\pi}\left(a_{i_{1} \ldots i_{d}}\right) \geq i_{1}+\cdots i_{d}$. Plus généralement, si $B$ est une $\mathcal{V}$-algèbre de type fini avec la présentation $B=\mathcal{V}\left[t_{1}, \ldots, t_{d}\right] / I$, où $I$ un idéal de $\mathcal{V}\left[t_{1}, \ldots, t_{d}\right]$, alors $B^{\dagger}=\mathcal{V}\left[t_{1}, \ldots, t_{d}\right]^{\dagger} / I \mathcal{V}\left[t_{1}, \ldots, t_{d}\right]^{\dagger}$. Une $\mathcal{V}$ algèbre f.c.t.f est une $\mathcal{V}$-algèbre commutative faiblement complète de type 
fini, i.e., un quotient de $\mathcal{V}\left[t_{1}, \ldots, t_{d}\right]^{\dagger}$. Un morphisme d'algèbres f.c.t.f. est une application entre deux $\mathcal{V}$-algèbres f.c.t.f. qui est un morphisme d'anneaux (unitaires).

Nous adopterons les notations suivantes :

Notation 1.2. Soit $B^{\dagger}$ une $\mathcal{V}$-algèbre f.c.t.f. On note $B_{0}=B^{\dagger} / \pi B^{\dagger}$ et, pour tout $f \in B^{\dagger}, \bar{f}$ désigne l'image de $f$ dans $B_{0}$. Pour tout $f \in B^{\dagger}$, on note $B^{\dagger}[f]$, la complétion faible de $\left(B^{\dagger}\right)_{f}$.

DÉfinition 1.3 (Schémas formels faibles affines). Meredith a introduit dans [Mer72] la notion de schéma formel faible affine associé à $B^{\dagger}$, noté $\operatorname{Spff}\left(B^{\dagger}\right)$. Il est défini comme l'espace annelé $\left(Y^{\dagger}, \mathcal{O}_{Y^{\dagger}}\right)$ dont l'espace topologique sous-jacent correspond à $Y^{\dagger}=Y_{0}=\operatorname{Spec} B_{0}$ (cet ensemble est aussi égal à celui des idéaux premiers ouverts de $B^{\dagger}$ ) et dont les sections du faisceau structural $\mathcal{O}_{Y^{\dagger}}$ sur un ouvert principal $Y_{0, \bar{f}}$ où $f \in B^{\dagger}$ est $\mathcal{O}_{Y^{\dagger}}\left(Y_{0, \bar{f}}\right):=B^{\dagger}[f]$ (on calcule que cet anneau ne dépend pas du choix du relèvement $f$ de $\bar{f}$ : voir [Mer72]).

ThÉorÈme 1.4 (Théorèmes $A$ et $B$ de Meredith). Soient $B^{\dagger}$ une $\mathcal{V}$-algèbre f.c.t.f. et $Y^{\dagger}:=\operatorname{Spff}\left(B^{\dagger}\right)$ le $\mathcal{V}$-schéma formel faible affine associé. Meredith a établi dans respectivement [Mer72, 3.3 et 2.14] :

1. Nous disposons du théorème de type $A$ : les foncteurs $M \mapsto \mathcal{O}_{Y^{\dagger}} \otimes_{B^{\dagger}} M$ et $\mathcal{M} \mapsto \Gamma\left(Y^{\dagger}, \mathcal{M}\right)$ sont des équivalences quasi-inverses entre la catégorie des $\mathcal{O}_{Y^{\dagger}}$-modules cohérents et celle des $B^{\dagger}$-modules de type fini.

2. Nous bénéficions aussi de théorème de type $B$ : pour tout $\mathcal{O}_{Y^{\dagger}}{ }^{-}$ module cohérent $\mathcal{M}$, pour tout entier $i>0, H^{i}\left(Y^{\dagger}, \mathcal{M}\right)=0$.

Ce théorème 1.4 reste valable après application du foncteur $\mathrm{Q} \otimes_{Z}-$, i.e. avec les $\mathcal{O}_{Y^{\dagger}, Q^{-}}$-modules cohérents à la place des $\mathcal{O}_{Y^{\dagger}}$-modules cohérents.

DÉFINITION 1.5. Un $\mathcal{V}$-schéma formel faible est un espace localement annelé en $\mathcal{V}$-algèbres $\left(Y^{\dagger}, \mathcal{O}_{Y^{\dagger}}\right)$ localement isomorphe à un $\mathcal{V}$-schéma formel faible affine (voir [Mer72]).

DÉfInition 1.6. Soit P estun $\mathcal{V}$-schéma de type fini. Meredith ([Mer72, 4]) construit le faisceau $\mathcal{O}_{P}^{\dagger}$ de la façon suivante : si $U \subset V \subset P$ sont des ouverts affines de $P$, alors $\Gamma\left(U, \mathcal{O}_{P}^{\dagger}\right):=\Gamma\left(U, \mathcal{O}_{P}\right)^{\dagger}$ et $\Gamma\left(V, \mathcal{O}_{P}^{\dagger}\right) \rightarrow \Gamma\left(U, \mathcal{O}_{P}^{\dagger}\right)$ 
est le morphisme canonique induit par complétion faible p-adique de $\Gamma\left(V, \mathcal{O}_{P}\right) \rightarrow \Gamma\left(U, \mathcal{O}_{P}\right)(\operatorname{voir}[\mathrm{Mer} 72,1.5])$. En notant $P^{\dagger}$ l'espace topologique de $P_{0}=P \times_{\text {Spec } \mathcal{V}} \operatorname{Spec}(k)$, il vérifie que l'espace annelé $\left(P^{\dagger}, \mathcal{O}_{P}^{\dagger}\right)$ est un $\mathcal{V}$ schéma formel faible et que l'on dispose d'un morphisme canonique

$$
\varepsilon:\left(P^{\dagger}, \mathcal{O}_{P}^{\dagger}\right) \rightarrow\left(P, O_{P}\right),
$$

qu'il appelle complétion faible de $\left(P, \mathcal{O}_{P}\right)$. Par abus de notation, on écrira $P^{\dagger}$ à la place de $\left(P^{\dagger}, \mathcal{O}_{P}^{\dagger}\right)$. On vérifie de plus que l'application $P \mapsto P^{\dagger}$ induit canoniquement (via [Mer72, 1.5]) un foncteur de la catégorie des $\mathcal{V}$-schémas de type fini dans celle des $\mathcal{V}$-schémas formels faibles.

EXemple 1.7. Donnons un exemple des constructions de 1.6. Lorsque $B$ est une $\mathcal{V}$-algèbre de type fini et $P:=\operatorname{Spec}(B)$ alors $P^{\dagger}=\operatorname{Spff}\left(B^{\dagger}\right)$. Dans ce cas, le morphisme $\varepsilon: P^{\dagger} \rightarrow P$ est topologiquement l'immersion fermée $\operatorname{Spec}\left(B_{0}\right) \hookrightarrow \operatorname{Spec}(B)$, le morphisme de faisceaux se déduit du morphisme canonique de $\mathcal{V}$-algèbres $B \rightarrow B^{\dagger}$. En générale, le morphisme $\varepsilon$ se construit par recollement.

\section{Isocristaux surconvergents sur $U_{0}$}

Comme $Z$ est un diviseur ample de $X$, nous pouvons dans notre contexte utiliser les théorèmes de type $A$ de Noot-Huyghe (voir [NH03]) pour les $\mathcal{D}_{\mathfrak{X}}^{\dagger}\left({ }^{\dagger} Z_{0}\right)_{Q}$-modules cohérents :

THÉORÈme 2.1 (Noot-Huyghe). 1. Les foncteurs sections globales $\Gamma(\mathfrak{X},-)$ et $\mathcal{D}_{\mathfrak{X}}^{\dagger}\left({ }^{\dagger} Z_{0}\right)_{\mathrm{Q}} \otimes_{\left.D_{\mathfrak{X}}^{\dagger} \dagger Z_{0}\right)_{\mathbb{Q}}}-$ induisent des équivalences quasi-inverses

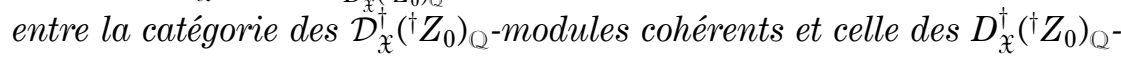
modules cohérents.

2. Les foncteurs sections globales $\Gamma(\mathfrak{X},-)$ et $\mathcal{D}_{\mathfrak{X}}\left({ }^{\dagger} Z_{0}\right)_{\mathbb{Q}} \otimes_{D_{\mathfrak{X}}\left({ }^{\dagger} Z_{0}\right)_{Q}}-$ induisent des équivalences quasi-inverses entre la catégorie des $\mathcal{D}_{\mathfrak{X}}\left({ }^{\dagger} Z_{0}\right)_{\mathrm{Q}^{-}}$ modules cohérents et celle des $D_{\mathfrak{X}}\left({ }^{\dagger} Z_{0}\right)_{\mathrm{Q}}$-modules cohérents.

3. Les foncteurs sections globales $\Gamma(\mathfrak{X},-)$ et $\mathcal{O}_{\mathfrak{X}}\left({ }^{\dagger} Z_{0}\right)_{\mathrm{Q}} \otimes_{O_{\mathfrak{X}}\left({ }^{\dagger} Z_{0}\right)_{\mathrm{Q}}}-$ induisent des équivalences quasi-inverses entre la catégorie des $\mathcal{O}_{\mathfrak{X}}\left({ }^{\dagger} Z_{0}\right)_{\mathrm{Q}^{-}}$ modules cohérents et celle des $O_{\mathfrak{X}}^{\dagger}\left(^{\dagger} Z_{0}\right)_{\mathrm{Q}}$-modules cohérents.

2.2 (Foncteur $\mathrm{sp}_{+}$). Soit $\mathcal{E}^{\dagger}$ un $\mathcal{D}_{U^{\dagger}, \mathrm{Q}}$-module cohérent, $\mathcal{O}_{U^{\dagger}, \mathrm{Q}}$-cohérent. On obtient un $\mathcal{D}_{\mathcal{X}}^{\dagger}\left({ }^{\dagger} Z_{0}\right)_{\mathrm{Q}}$-module cohérent en posant

$$
\mathrm{sp}_{+}\left(\mathcal{E}^{\dagger}\right):=\mathcal{D}_{\mathfrak{X}}^{\dagger}\left({ }^{\dagger} Z_{0}\right)_{\mathrm{Q}} \otimes_{j_{0 *} \mathcal{D}_{U^{\dagger}, \mathrm{Q}}} j_{0 *}\left(\mathcal{E}^{\dagger}\right) .
$$

Ce foncteur a été défini dans [Car06a] dans un contexte un peu plus général. 
Nous redémontrons et surtout affinons (via l'isomorphisme 2.5.1 qui nous sera utile) dans notre contexte (plus facile) les résultats analogues avec structure de Frobenius de [Car07] ou sans structure de Frobenius de [Car11b].

2.3 (Catégories d'isocristaux surconvergents sur $U_{0}$ ).

- On note Isoc ${ }^{\dagger}\left(A_{K}^{\dagger}\right)$ la catégorie des $A_{K}^{\dagger}$-modules cohérents munis d'une connexion surconvergente. D'après [Ber96a, 5], cette catégorie est canoniquement isomorphe à celle des isocristaux surconvergents sur $U_{0}$.

- On note $\operatorname{Isoc}^{\dagger}\left(\mathcal{O}_{U^{\dagger}, Q}\right)$ la catégorie des $\mathcal{D}_{U^{\dagger}, Q^{-}}$-modules cohérents, $\mathcal{O}_{U^{\dagger}, Q}$-cohérents tels que le $A_{K}^{\dagger}$-module cohérent correspondant via le théorème $A$ de Meredith (voir 1.4) soit un élément de $\operatorname{Isoc}^{\dagger}\left(A_{K}^{\dagger}\right)$.

- On note Isoc ${ }^{\dagger}\left(\mathfrak{X}, Z_{0} / K\right)$ la catégorie des $\mathcal{D}_{\mathfrak{X}}^{\dagger}\left({ }^{\dagger} Z_{0}\right)_{\mathrm{Q}}$-modules cohérents, $\mathcal{O}_{\mathfrak{X}}\left({ }^{\dagger} Z_{0}\right)_{\mathbb{Q}}$-cohérents. On rappelle que pour tout $\mathcal{G} \in \operatorname{Isoc}^{\dagger}\left(\mathfrak{X}, Z_{0} / K\right), \mathcal{G}$ est $\mathcal{D}_{\mathfrak{X}}\left({ }^{\dagger} Z_{0}\right)_{\mathrm{Q}}$-cohérent et le morphisme canonique $\mathcal{D}_{\mathfrak{X}}^{\dagger}\left({ }^{\dagger} Z_{0}\right)_{\mathrm{Q}} \otimes_{\mathcal{D}_{\mathfrak{X}}\left({ }^{\dagger} Z_{0}\right)_{Q}} \mathcal{G} \rightarrow \mathcal{G}$ est un isomorphisme (voir [Car05, 2.2.8]). De plus, la catégorie $\operatorname{Isoc}^{\dagger}\left(\mathfrak{X}, Z_{0} / K\right)$ est canoniquement (via les foncteurs quasi-inverses image directe $\mathrm{sp}_{*}$ et image inverse $\mathrm{sp}^{*}$ par le morphisme de spécialisation de $\mathrm{sp}: \mathfrak{X}_{K} \rightarrow \mathfrak{X}$ ) isomorphe à celle des isocristaux surconvergents sur $U_{0} / K$.

2.4. On déduit des théorèmes de type $A$ de 1.4 ou 2.1 les équivalences :

- Les foncteurs sections globales $\Gamma\left(U^{\dagger},-\right)$ et $\mathcal{D}_{U^{\dagger, Q}} \otimes_{D_{U, Q}}-$ (ou $\left.\mathcal{O}_{U^{\dagger, Q}} \otimes_{A_{Q}}-\right)$ induisent des équivalences quasi-inverses entre $\operatorname{Iscc}^{\dagger}\left(A_{K}^{\dagger}\right)$ et $\operatorname{Isoc}^{\dagger}\left(\mathcal{O}_{U^{\dagger}, \mathrm{Q}}\right)$.

- Les foncteurs sections globales $\Gamma(\mathfrak{X},-)$ et $\mathcal{D}_{\mathfrak{X}}^{\dagger}\left({ }^{\dagger} Z_{0}\right)_{\mathrm{Q}} \otimes_{D_{\mathfrak{X}}^{\dagger}\left(Z_{0}\right)_{\mathrm{Q}}}-(\mathrm{ou}$ $\mathcal{D}_{\mathfrak{X}}\left({ }^{\dagger} Z_{0}\right)_{\mathrm{Q}} \otimes_{D_{\mathfrak{X}}\left({ }^{\dagger} Z_{0}\right)_{Q}}-$ ou $\left.\mathcal{O}_{\mathfrak{X}}\left({ }^{\dagger} Z_{0}\right)_{\mathrm{Q}} \otimes_{A_{K}^{\dagger}}-\right)$ induisent des équivalences quasi-inverses entre $\operatorname{Isoc}^{\dagger}\left(A_{K}^{\dagger}\right)$ et $\operatorname{Isoc}^{\dagger}\left(\mathfrak{X}, Z_{0} / K\right)$.

Proposition 2.5. Le foncteur $\mathrm{sp}_{+}$se factorise en une équivalence de catégories de la forme $\operatorname{sp}_{+}$: $\operatorname{Isoc}^{\dagger}\left(\mathcal{O}_{U^{\dagger}, \mathrm{Q}}\right) \cong \operatorname{Isoc}^{\dagger}\left(\mathfrak{X}, Z_{0} / K\right)$. Pour tout $\mathcal{E}^{\dagger} \in \operatorname{Isoc}^{\dagger}\left(\mathcal{O}_{U^{\dagger}, Q}\right)$, on dispose en outre de l'isomorphisme canonique :

$$
\Gamma\left(\mathfrak{X}, \mathrm{sp}_{+}\left(\mathcal{E}^{\dagger}\right)\right) \stackrel{\sim}{\longrightarrow} \Gamma\left(U^{\dagger}, \mathcal{E}^{\dagger}\right) .
$$

DÉmonstration. Soit $\mathcal{E}^{\dagger} \in \operatorname{Isoc}^{\dagger}\left(\mathcal{O}_{U^{\dagger}, Q}\right)$. D'après le premier point de 2.4, $E^{\dagger}:=\Gamma\left(U^{\dagger}, \mathcal{E}^{\dagger}\right) \in \operatorname{Isoc}^{\dagger}\left(A_{K}^{\dagger}\right)$ et le morphisme canonique $\mathcal{D}_{U^{\dagger}, \mathrm{Q}} \otimes_{D_{U^{\dagger}, Q}} E^{\dagger} \rightarrow \mathcal{E}^{\dagger}$ est un isomorphisme. En choisissant une présentation finie de $E^{\dagger}$ par des $D_{U^{\dagger}, Q}$-modules libres, via l'exactitude du foncteur $j_{0 *}$ sur la catégorie des $D_{U^{\dagger}, Q}$-modules globalement de présentation 
finie (voir [Car06a, 2.2.9.1]), il résulte du lemme des cinq que l'on bénéficie de l'isomorphisme canonique $j_{0 *} \mathcal{D}_{U^{\dagger}, \mathrm{Q}} \otimes_{D_{U^{\dagger}, \mathrm{Q}}} E^{\dagger} \stackrel{\sim}{\sim} j_{0 *}\left(\mathcal{D}_{U^{\dagger}, \mathrm{Q}} \otimes_{D_{U^{\dagger}, \mathrm{Q}}} E^{\dagger}\right)$. Comme $j_{0 *}\left(\mathcal{D}_{U^{\dagger}, \mathrm{Q}} \otimes_{D_{U^{\dagger}, Q}} E^{\dagger}\right) \rightarrow j_{0 *}\left(\mathcal{E}^{\dagger}\right)$, on en déduit l'isomorphisme $j_{0 *} \mathcal{D}_{U^{\dagger}, Q} \otimes_{D_{U^{\dagger}, Q}} E^{\dagger} \stackrel{\sim}{\longrightarrow} j_{0 *}\left(\mathcal{E}^{\dagger}\right)$. Comme on dispose d'après Noot-Huyghe de la formule $D_{\mathfrak{X}}\left({ }^{\dagger} Z_{0}\right)_{\mathrm{Q}}=D_{U^{\dagger}, \mathrm{Q}}$ (voir [NH03]), il en résulte l'isomorphisme

$$
\mathrm{sp}_{+}\left(\mathcal{E}^{\dagger}\right) \stackrel{\sim}{\longrightarrow} \mathcal{D}_{\mathfrak{X}}^{\dagger}\left({ }^{\dagger} Z_{0}\right)_{\mathrm{Q}} \otimes_{D_{U^{\dagger}, \mathrm{Q}}} E^{\dagger}=\mathcal{D}_{\mathfrak{X}}^{\dagger}\left({ }^{\dagger} Z_{0}\right)_{\mathrm{Q}} \otimes_{D_{\mathfrak{X}}\left({ }^{\dagger} Z_{0}\right)_{\mathrm{Q}}} E^{\dagger} .
$$

D'après les équivalences de catégories de 2.4, on vérifie $\mathcal{G}:=\mathcal{D}_{\mathfrak{X}}\left({ }^{\dagger} Z_{0}\right)_{\mathrm{Q}} \otimes_{D_{\mathfrak{X}}\left({ }^{\dagger} Z_{0}\right)_{\mathrm{Q}}} E^{\dagger} \in \operatorname{Isoc}^{\dagger}\left(\mathfrak{X}, Z_{0} / K\right)$. Il en résulte que le morphisme canonique $\mathcal{D}_{\mathfrak{X}}^{\dagger}\left({ }^{\dagger} Z_{0}\right)_{\mathrm{Q}} \otimes_{\left.\mathcal{D}_{\mathfrak{X}}{ }^{\dagger} Z_{0}\right)_{\mathrm{Q}}} \mathcal{G} \rightarrow \mathcal{G}$ est un isomorphisme (voir [Car05, 2.2.8]). On en déduit l'isomorphisme :

$$
\mathcal{D}_{\mathfrak{X}}^{\dagger}\left({ }^{\dagger} Z_{0}\right)_{\mathrm{Q}} \otimes_{D_{\mathfrak{X}}\left(Z_{0}\right)_{\mathrm{Q}}} E^{\dagger} \stackrel{\sim}{\longrightarrow} \mathcal{D}_{\mathfrak{X}}\left({ }^{\dagger} Z_{0}\right)_{\mathrm{Q}} \otimes_{D_{\mathfrak{X}}\left({ }^{\dagger} Z_{0}\right)_{\mathrm{Q}}} E^{\dagger} .
$$

Avec deux équivalences de catégorie du deuxième point de 2.4, on obtient l'isomorphisme dans $\operatorname{Isoc}^{\dagger}\left(\mathfrak{X}, Z_{0} / K\right)$ :

$$
\mathcal{D}_{\mathfrak{X}}\left({ }^{\dagger} Z_{0}\right)_{Q} \otimes_{D_{\mathfrak{X}}\left({ }^{\dagger} Z_{0}\right)_{\mathrm{Q}}} E^{\dagger} \stackrel{\sim}{\longrightarrow} \mathcal{D}_{\mathfrak{X}}^{\dagger}\left({ }^{\dagger} Z_{0}\right)_{\mathrm{Q}} \otimes_{D_{\mathfrak{X}}^{\dagger}\left({ }^{\dagger} Z_{0}\right)_{Q}} E^{\dagger} .
$$

En composant ces trois isomorphismes, on obtient l'isomorphisme qui nous permet de conclure :

$$
\operatorname{sp}_{+}\left(\mathcal{E}^{\dagger}\right) \stackrel{\sim}{\longrightarrow} \mathcal{D}_{\mathfrak{X}}^{\dagger}\left({ }^{\dagger} Z_{0}\right)_{Q} \otimes_{D_{\mathfrak{X}}^{\dagger}\left(Z_{0}\right)_{Q}} E^{\dagger}
$$

REMARque 2.6. La catégorie $\operatorname{Isoc}^{\dagger}\left(\mathcal{O}_{U^{\dagger}, \mathrm{Q}}\right)$ est égale à la catégorie de Christol et Mebkhout notée $\operatorname{MLS}\left(\mathcal{O}_{\mathrm{U}^{\dagger} / \mathrm{K}}\right)$ dans [CM01] des $\mathcal{O}_{U^{\dagger} / K^{-m o d u l e s}}$ localement libres de rang fini à connexion et qui soient solubles dans les classes singulières de $Z_{0}$ (voir l'introduction de [CM01] à la page 630). Le théorème 2.5 fait donc le lien entre les modules solubles utilisés par Christol-Mebkhout dans leurs théorèmes de [CM01] et les $\mathcal{D}$-modules arithmétiques de Berthelot qui sont des isocristaux surconvergents.

\section{Foncteur daguification}

3.1. On note $\operatorname{MC}\left(\mathcal{O}_{U^{\dagger}, \mathrm{Q}}\right)$ la catégorie des $\mathcal{D}_{U^{\dagger}, \mathrm{Q}}$-modules cohérents, $\mathcal{O}_{U^{\dagger}, \mathrm{Q}}$-cohérents ; $\mathrm{MC}\left(\mathcal{O}_{U, \mathrm{Q}}\right)$ la catégorie des $\mathcal{D}_{U, \mathrm{Q}}$-modules cohérents, $\mathcal{O}_{U, Q}$-cohérents. On dispose du foncteur canonique :

$$
\dagger: \operatorname{MC}\left(\mathcal{O}_{U, \mathrm{Q}}\right) \rightarrow \operatorname{MC}\left(\mathcal{O}_{U^{\dagger}, \mathrm{Q}}\right)
$$


défini par $\mathcal{E} \mapsto \mathcal{E}^{\dagger}:=\mathcal{O}_{U^{\dagger}, \mathrm{Q}} \otimes_{\varepsilon^{-1} \mathcal{O}_{U, Q}} \varepsilon^{-1} \mathcal{E}$. Ce foncteur est bien défini d'après le paragraphe 3.3 (plus précisément 3.3.2). Dans la suite de ce chapitre, on se fixe $\mathcal{E} \in \mathrm{MC}\left(\mathcal{O}_{U^{\dagger}, \mathrm{Q}}\right)$ et on note $\mathcal{E}^{\dagger}$ son image par le foncteur 3.1.1. On pose aussi $E:=\Gamma(U, \mathcal{E})$ et $E^{\dagger}:=\Gamma\left(U^{\dagger}, \mathcal{E}^{\dagger}\right)$.

Énonçons le théorème de type $A$ et $B$ sous sa forme algébrique :

ThÉORÈme 3.2 (Théorèmes $A$ et $B$ ). Soient $Y$ un $\mathcal{V}$-schéma affine de type fini et $Y_{K}:=Y \otimes_{\mathcal{V}} K$.

1. Les foncteurs $M \mapsto \mathcal{O}_{Y, Q} \otimes_{O_{Y, Q}} M$ et $\mathcal{M} \mapsto \Gamma(Y, \mathcal{M})$ sont des équivalences quasi-inverses entre la catégorie des $\mathcal{O}_{Y, Q}$-modules cohérents et celle des $O_{Y, \mathrm{Q}}$-modules de type fini.

2. Les foncteurs $M \mapsto \mathcal{D}_{Y, \mathrm{Q}} \otimes_{D_{Y, \mathrm{Q}}} M$ et $\mathcal{M} \mapsto \Gamma(Y, \mathcal{M})$ sont des équivalences quasi-inverses entre la catégorie des $\mathcal{D}_{Y, \mathrm{Q}}$-modules cohérents et celle des $D_{Y, \mathrm{Q}}$-modules de type fini.

3. Les foncteurs $M \mapsto \mathcal{D}_{Y_{K}} \otimes_{D_{Y_{K}}} M$ et $\mathcal{M} \mapsto \Gamma\left(Y_{K}, \mathcal{M}\right)$ sont des équivalences quasi-inverses entre la catégorie des $\mathcal{D}_{Y_{K}}$-modules cohérents et celle des $D_{Y_{K}}$-modules de type fini.

4. Pour tout $\mathcal{O}_{Y, Q}$-module cohérent $\mathcal{M}$ (resp. $\mathcal{D}_{Y, \mathrm{Q}}$-module cohérent $\mathcal{M})$, pour tout entier $i>0, H^{i}(Y, \mathcal{M})=0$. Pour tout $\mathcal{D}_{Y_{K}}$-module cohérent $\mathcal{M}$, pour tout entier $i>0, H^{i}\left(Y_{K}, \mathcal{M}\right)=0$.

3.3 (Description du foncteur $\dagger$ ). D'après les théorèmes de type $A$ sur les $\mathcal{O}_{U, Q}$-modules ou $\mathcal{D}_{U, Q}$-modules cohérents (voir 3.2), on dispose des isomorphismes canoniques :

$$
\mathcal{O}_{U, \mathrm{Q}} \otimes_{A_{\mathrm{Q}}} E \stackrel{\sim}{\longrightarrow} \mathcal{D}_{U, \mathrm{Q}} \otimes_{D_{U, \mathrm{Q}}} E \stackrel{\sim}{\longrightarrow} \mathcal{E} .
$$

Comme le morphisme canonique $\mathcal{O}_{U^{\dagger, \mathrm{Q}}} \otimes_{\varepsilon^{-1} \mathcal{O}_{U, \mathrm{Q}}} \varepsilon^{-1} \mathcal{D}_{U, \mathrm{Q}} \rightarrow \mathcal{D}_{U^{\dagger}, \mathrm{Q}}$ est un isomorphisme, on en déduit les isomorphismes canoniques :

$$
\mathcal{O}_{U^{\dagger}, \mathrm{Q}} \otimes_{A_{\mathrm{Q}}} E \stackrel{\sim}{\longrightarrow} \mathcal{D}_{U^{\dagger}, \mathrm{Q}} \otimes_{D_{U, \mathrm{Q}}} E \stackrel{\sim}{\longrightarrow} \mathcal{E}^{\dagger} .
$$

Via le théorème de type $A$ sur les $\mathcal{D}_{U^{\dagger}, Q^{-}}$-modules cohérents, il en dérive $D_{U^{\dagger}, \mathrm{Q}} \otimes_{D_{U, Q}} E \stackrel{\sim}{\longrightarrow} E^{\dagger}$.

3.4 (Holonomie sur $Y_{K}$ ). Soit $Y_{K}$ une variété sur $K$. Comme $K$ est de caractéristique nulle, on dispose de la notion de $\mathcal{D}_{Y_{K}}$-module holonome. Cette notion est stable par les six opérations de Grothendieck. Par définition, un $\mathcal{D}_{Y_{K}}$-module cohérent est holonome lorsque sa variété car- 
actéristique est de dimension minimale (l'inégalité de Bernstein donne une minoration). On dispose de plus de la caractérisation suivante de l'holonomie : un $\mathcal{D}_{Y_{K}}$-module cohérent $\mathcal{G}$ est holonome si et seulement si, pour tout $l \neq \operatorname{dim} Y_{K}, \mathcal{E} t_{\mathcal{D}_{Y_{K}}}^{l}\left(\mathcal{G}, \mathcal{D}_{Y_{K}}\right)=0$.

Par exemple, un $\mathcal{D}_{Y_{K}}$-module cohérent qui est $\mathcal{O}_{Y_{K}}$-cohérent est holonome. Plus généralement, si $Y_{K}$ est une courbe alors un $\mathcal{D}_{Y_{K}}$-module cohérent $\mathcal{F}$ est holonome si et seulement si $\mathcal{F}$ est $\mathcal{O}_{Y_{K}}$-cohérent sur un ouvert dense de $Y_{K}$.

Lemme 3.5. Le $\mathcal{D}_{X, \mathrm{Q}}$-module $j_{*}(\mathcal{E})$ est cohérent. De plus, pour tout $l \neq d_{X_{0}}, \mathcal{E}_{x} t_{\mathcal{D}_{X, Q}}^{l}\left(j_{*}(\mathcal{E}), \mathcal{D}_{X, Q}\right)=0$.

DÉmonstration. L'assertion est locale en $X$. On peut supposer $X$ affine, muni de coordonnées locales et $Z$ défini par une équation locale. D'après le théorème de type $A$ sur les $\mathcal{D}_{U, \mathrm{Q}}$-modules cohérents (resp. les $\mathcal{O}_{U, \mathrm{Q}}$-modules cohérents) énoncé dans 3.2, $E:=\Gamma(U, \mathcal{E})$ est un $D_{U, \mathrm{Q}}$-module cohérent (resp. $O_{U, \mathrm{Q}}$-module cohérent). Or, $\Gamma\left(U, \mathcal{D}_{U, \mathrm{Q}}\right)=\Gamma\left(U_{K}, \mathcal{D}_{U_{K}}\right)$ et $\Gamma\left(U, \mathcal{O}_{U, \mathrm{Q}}\right)=$ $\Gamma\left(U_{K}, \mathcal{O}_{U_{K}}\right)$. On note $\mathcal{F}:=\mathcal{D}_{U_{K}} \otimes_{\Gamma\left(U_{K}, \mathcal{D}_{U_{K}}\right)} E \stackrel{\sim}{\longleftarrow} \mathcal{O}_{U_{K}} \otimes_{\Gamma\left(U_{K}, \mathcal{O}_{U_{K}}\right)} E$ le $\mathcal{D}_{U_{K}}-$ module cohérent, $\mathcal{O}_{U_{K}}$-cohérent correspondant.

Comme $U_{K}$ est une variété sur $K$ qui est de caractéristique nulle, comme $\mathcal{F}$ est un $\mathcal{D}_{U_{K}}$-module holonome (car $\mathcal{O}_{U_{K}}$-cohérent), alors par préservation de l'holonomie par image directe, $j_{K *}(\mathcal{F})$ est une $\mathcal{D}_{X_{K}}$-module holonome. Comme l'entier $d_{X_{0}}$ est aussi la dimension de $X_{K}$, d'après les théorèmes de type $A$ et $B$, il en résulte que $\Gamma\left(X_{K}, j_{K *}(\mathcal{F})\right)$ est un $D_{X_{K}}$-module cohérent et que, pour tout $l \neq d_{X_{0}}, \operatorname{Ext}_{D_{X_{K}}}^{l}\left(\Gamma\left(X_{K}, j_{K *}(\mathcal{F})\right), D_{X_{K}}\right)=0$. Comme $D_{X, \mathrm{Q}}=$ $=D_{X_{K}}$ et $\Gamma\left(X, j_{*}(\mathcal{E})\right)=\Gamma\left(X_{K}, j_{K *}(\mathcal{F})\right)$, alors $\Gamma\left(X, j_{*}(\mathcal{E})\right)$ est $D_{X, \mathrm{Q}}$-cohérent. La quasi-cohérence de $j_{*}(\mathcal{E})$ nous permet alors d'en conclure que $j_{*}(\mathcal{E})$ est $\mathcal{D}_{X, Q}$-cohérent. Via les théorèmes de type $A$ et $B$, il en résulte que $\mathcal{E}_{x} t_{\mathcal{D}_{X, Q}}^{l}\left(j_{*}(\mathcal{E}), \mathcal{D}_{X, \mathrm{Q}}\right)=0$ si et seulement si $\operatorname{Ext}_{D_{X, Q}}^{l}\left(\Gamma\left(X, j_{*}(\mathcal{E})\right), D_{X, Q}\right)=0$. D'où le résultat.

3.6. Grâce au lemme 3.5, on obtient un $\mathcal{D}_{X^{\dagger}, Q}$-module cohérent en posant

$$
j_{*}^{\dagger}(\mathcal{E}):=\mathcal{D}_{X^{\dagger}, Q} \otimes_{\varepsilon^{-1} \mathcal{D}_{X, Q}} \varepsilon^{-1}\left(j_{*} \mathcal{E}\right) .
$$

DÉfinition 3.7 (Holonomie). De manière analogue au cas algébrique (voir 3.4), on définit dans [Car11a] la notion d'holonomie de la manière suivante : un $\mathcal{D}_{\mathfrak{X}, \mathrm{Q}}^{\dagger}$-module $\mathcal{G}$ est holonome s'il est $\mathcal{D}_{\mathfrak{X}, \mathrm{Q}}^{\dagger}$-cohérent et si, pour tout entier $l \neq d_{X_{0}}, \mathcal{E} x t_{\mathcal{D}_{x, Q}^{\dagger}}^{l}\left(\mathcal{G}, \mathcal{D}_{\mathfrak{X}, Q}^{\dagger}\right)=0$. Contrairement à la version al- 
gébrique de 3.4, on ne sait toujours pas construire canoniquement une variété caractéristique associée à $\mathcal{G}$ (sauf d'après Berthelot lorsque le $\mathcal{G}$ est muni d'une structure de Frobenius : voir [Ber02]). Lorsque l'on dispose d'une structure de Frobenius, on retrouve la définition de Berthelot.

EXEMPLE 3.8. • Un $\mathcal{D}_{\mathfrak{X}, \mathrm{Q}}^{\dagger}$-module cohérent qui est $\mathcal{O}_{\mathfrak{X}, \mathrm{Q}}$-cohérent (i.e. un isocristal convergent sur $X$ ) est holonome.

- Si $X_{0}$ est de dimension pure égale à 1 , un $\mathcal{D}_{\mathfrak{X}, \mathrm{Q}}^{\dagger}$-module cohérent $\mathcal{G}$ est holonome si $\mathcal{H o m}_{\mathcal{D}_{\mathfrak{X}, Q}^{\dagger}}\left(\mathcal{G}, \mathcal{D}_{\mathfrak{X}, \mathrm{Q}}^{\dagger}\right)=0$.

LEMme 3.9. Avec les notations de 3.6, le $\mathcal{D}_{\mathfrak{X}, \mathrm{Q}}^{\dagger}$-module $\mathcal{D}_{\mathfrak{X}, \mathrm{Q}}^{\dagger} \otimes_{\mathcal{D}_{X^{\dagger}, \mathrm{Q}}} j_{*}^{\dagger}(\mathcal{E})$ est holonome.

DÉmonstration. La $\mathcal{D}_{\mathfrak{X}, \mathrm{Q}}^{\dagger}$-cohérence de $\mathcal{G}:=\mathcal{D}_{\mathfrak{X}, \mathrm{Q}}^{\dagger} \otimes_{\mathcal{D}_{X \dagger, \mathrm{Q}}} j_{*}^{\dagger}(\mathcal{E})$ se déduit $d u$ lemme 3.5. Soit $l \neq d_{X_{0}}$ un entier. Pour vérifier que $\mathcal{E} x t_{\mathcal{D}_{\mathfrak{X}, Q}^{\dagger}}^{l}\left(\mathcal{D}_{\mathfrak{X}, Q}^{\dagger} \otimes_{\mathcal{D}_{X \dagger, Q}} j_{*}^{\dagger}(\mathcal{E}), \mathcal{D}_{\mathfrak{X}, \mathrm{Q}}^{\dagger}\right)=0$, on se ramène au cas où $X$ est affine. Comme l'extension $D_{X, Q} \rightarrow D_{\ngtr}^{\dagger}\left({ }^{\dagger} Z_{0}\right)_{Q}$ est plate, cela résulte alors de 3.5.

LEMME 3.10. Avec les notations de 3.6, on dispose de l'isomorphisme canonique de $\mathcal{D}_{\mathfrak{X}}^{\dagger}\left({ }^{\dagger} Z_{0}\right)_{\mathrm{Q}}$-modules cohérents :

$$
\mathcal{D}_{\mathfrak{X}}^{\dagger}\left({ }^{\dagger} Z_{0}\right)_{Q} \otimes_{\mathcal{D}_{X^{\dagger}, Q}} j_{*}^{\dagger}(\mathcal{E}) \stackrel{\sim}{\longrightarrow} \operatorname{sp}_{+}\left(\mathcal{E}^{\dagger}\right) .
$$

DÉmonstration. Comme le morphisme canonique $\mathcal{O}_{U^{\dagger, Q}} \otimes_{\varepsilon^{-1} \mathcal{O}_{U, Q}}$. $\varepsilon^{-1} \mathcal{D}_{U, Q} \rightarrow \mathcal{D}_{U^{\dagger, Q}}$ est un isomorphisme, on vérifie que le morphisme canonique $\mathcal{E}^{\dagger} \rightarrow \mathcal{D}_{U^{\dagger}, Q} \otimes_{\varepsilon^{-1}} \mathcal{D}_{U, Q} \varepsilon^{-1}(\mathcal{E})$ est un isomorphisme. Il en résulte les isomorphismes $\mathcal{D}_{U^{\dagger}, \mathrm{Q}}$-linéaires :

$$
j_{0}^{-1}\left(j_{*}^{\dagger}(\mathcal{E})\right) \stackrel{\sim}{\longrightarrow} \mathcal{D}_{U^{\dagger}, \mathrm{Q}} \otimes_{\mathcal{E}^{-1} \mathcal{D}_{U, \mathrm{Q}}} \varepsilon^{-1}(\mathcal{E}) \stackrel{\sim}{\longleftarrow} \mathcal{E}^{\dagger} .
$$

Comme $j_{0 *}$ est adjoint à droite de $j_{0}^{-1}$, on obtient alors le premier morphisme $j_{0 *} \mathcal{D}_{U^{\dagger}, \mathrm{Q}}$-linéaire $j_{*}^{\dagger}(\mathcal{E}) \rightarrow j_{0 *}\left(\mathcal{E}^{\dagger}\right) \rightarrow \mathrm{sp}_{+}\left(\mathcal{E}^{\dagger}\right)$. On en déduit par extension le morphisme 3.10.1 voulu. Enfin, comme la flèche 3.10 .1 est un morphisme de $\mathcal{D}_{\mathfrak{X}}^{\dagger}\left({ }^{\dagger} Z_{0}\right)_{\mathrm{Q}}$-modules cohérents qui est un isomorphisme en dehors de $Z$, celle-ci est bien un isomorphisme (voir [Ber96b, 4.3.12]).

\section{Conditions $(D N L-N L)$ et $(N L-N L)$}

Afin d'utiliser les définitions et résultats de Christol et Mebkhout, nous supposons dans cette section que $X_{0}$ est de dimension pure égale à 1 . Nous 
rappelons dans cette section les conditions $(N L)$ et $(D N L)$ qui apparaissent dans les travaux de Christol-Mebkhout dans [CM97, CM00, CM01].

4.1 (Nombres non Liouville). Rappelons qu'un nombre $\alpha \in Z_{p}$ a la propriété $(N L)$ (i.e. de non Liouvillité) si les séries

$$
\sum_{i \geq 0, i \neq \alpha} \frac{x^{i}}{i-\alpha}, \quad \sum_{i \geq 0, i \neq \alpha} \frac{x^{i}}{i+\alpha}
$$

ont un rayon de convergence égal à 1 . Par exemple, on sait que les entiers $p$-adiques algébriques sur Q ont la propriété $(N L)$. On remarque que cette propriété ne dépend que de la classe de $\alpha$ dans $Z_{p} / Z$. Cette propriété est intimement liée à la finitude cohomologique : un nombre $\alpha$ a la propriété $(N L)$ si et seulement si l'opérateur $x \frac{d}{d x}-\alpha$ a un indice sur l'espace $\mathcal{R}_{K}$ (voir aussi le deuxième point de 4.7).

4.2 (Propriétés $(D N L)$ et $(N L)$ de Christol-Mebkhout). Soit $\mathcal{M}$ un module sur $\mathcal{R}_{K}$ muni d'une connexion intégrable soluble en 1 (voir [CM00, 4.1.]). De tels $\mathcal{R}_{K}$-modules se construisent par exemple par extension à partir d'un isocristal surconvergent (voir 4.3).

- On dit que $\mathcal{M}$ a la propriété $(D N L)$ si l'exposant de la partie de pente nulle de $\mathcal{M}$ (la notion d'exposant d'un module de pente nulle est l'objet de [CM97]) a des différences qui ont la propriété non Liouville ( $N L)$ (voir 4.1). Dans ce cas, les exposants $p$-adiques de $\mathcal{M}$ sont des éléments de $Z_{p} / Z$ bien définis.

- On dit que $\mathcal{M}$ a la propriété $(N L)$ si $\mathcal{M}$ a la propriété $(D N L)$ et si ses exposants ont la propriété $(N L)$.

DÉfinition 4.3 Soit $\mathcal{E}^{\dagger} \in \operatorname{Isoc}^{\dagger}\left(\mathcal{O}_{U^{\dagger}, \mathrm{Q}}\right)$. Si $x$ est un point fermé de $Z_{k}$ rationnel sur $k$, alors $] x[x$ est isomorphe au disque unité ouvert. Il en résulte que $\mathcal{R}_{K}=\Gamma(] x\left[\mathfrak{x}, j_{0}^{\dagger} \mathcal{O}_{\mathfrak{X}_{K}}\right)$. Plus généralement, tout point fermé $x$ de $Z_{k}$ donne après extension finie $\mathcal{V} \rightarrow \mathcal{V}^{\prime}$ à $\operatorname{deg}(x)$ point $x^{\prime}$ rationnels sur $k^{\prime}$. En ajoutant des primes pour signifier tout changement de bases via cette extension finie $\mathcal{V} \rightarrow \mathcal{V}^{\prime}$, le $\mathcal{R}_{K}$-module associé à $\mathcal{E}^{\dagger}$ en $x^{\prime}$, i.e. $\mathcal{E}_{x^{\prime}}^{\dagger}:=$ $\Gamma(] x^{\prime}\left[\mathfrak{X}_{x^{\prime}}, j_{0}^{\prime \prime} \mathcal{O}_{\mathfrak{X}_{K^{\prime}}^{\prime}}\right) \otimes_{\Gamma\left(\mathfrak{X}_{K}, j^{\dagger} \mathcal{O}_{\mathfrak{X}_{K}}\right)} \Gamma\left(U^{\dagger}, \mathcal{E}^{\dagger}\right)$ ne dépend pas, à isomorphisme près, du choix d'un tel $x^{\prime}$. On le note $\mathcal{E}_{x}^{\dagger}$. Comme la connexion de $\mathcal{E}^{\dagger}$ est surconvergente, la connexion intégrable de $\mathcal{E}_{x}^{\dagger}$ induite est soluble en 1 .

- On dira que $\mathcal{E}^{\dagger}$ vérifie la propriété $(D N L-N L)$ si, pour tout point fermé $x$ de $X_{k}, \mathcal{E}_{x}^{\dagger}$ a la propriété $(D N L)$ et $\left.\operatorname{End}_{\mathcal{R}_{K}}\left(\mathcal{E}_{x}^{\dagger}\right)_{>0}\right)$ a la propriété $(N L)$. 
- On dira que $\mathcal{E}^{\dagger}$ vérifie la propriété $(N L-N L)$ si, pour tout point fermé $x$ de $X_{k}, \mathcal{E}_{x}^{\dagger}$ a la propriété $(N L)$ et $\operatorname{End}_{\mathcal{R}_{K}}\left(\left(\mathcal{E}_{x}^{\dagger}\right)_{>0}\right)$ a la propriété $(N L)$.

- On dira qu'un objet de Isoc ${ }^{\dagger}\left(\mathfrak{X}, Z_{0} / K\right)$ vérifie la propriété $(D N L-N L)$ (resp. $(N L-N L)$ ) si l'objet (à isomorphisme près) de $\operatorname{Isoc}^{\dagger}\left(\mathcal{O}_{U^{\dagger}, \mathrm{Q}}\right)$ correspondant via l'équivalence $\operatorname{sp}_{+}: \operatorname{Isoc}^{\dagger}\left(\mathcal{O}_{U^{\dagger}, Q}\right) \cong \operatorname{Isoc}^{\dagger}\left(\mathfrak{X}, Z_{0} / K\right)$ de 2.5 vérifie la propriété $(D N L-N L)$ (resp. $(N L-N L))$.

On remarquera que la propriété $(N L-N L)$ est plus forte que $(D N L-N L)$.

EXEMPLE 4.4. Soit $\mathcal{E}^{\dagger} \in \operatorname{Isoc}^{\dagger}\left(\mathcal{O}_{U^{\dagger}, Q}\right)$. Si $\mathcal{E}^{\dagger}$ est muni d'une structure de Frobenius, e.g. en prenant le faisceau trivial $\mathcal{O}_{U^{\dagger}, \mathrm{Q}}$ muni de la connexion triviale, alors $\mathcal{E}^{\dagger}$ vérifie la propriété $(N L-N L)$. En effet, on peut par exemple consulter la preuve de [CM01, 5.0-12] pour s'en convaincre.

THÉoRÈme 4.5 (Théorèmes d'algébrisation et de finitude de ChristolMebkhout). On dispose des théorèmes de Christol et Mebkhout (voir $[\mathrm{CM} 01,5]$ :

1. Si $\mathcal{E}^{\dagger}$ vérifie la propriété $(D N L-N L)$ alors, quitte à faire une extension finie du corps de base $K, \mathcal{E}^{\dagger}$ est dans l'image essentielle du foncteur $\dagger$ de 3.1.1.

2. Si $\mathcal{E}^{\dagger}$ vérifie la propriété $(N L-N L)$ alors les $K$-espaces de cohomologie de de Rham p-adique

$$
\operatorname{Hom}_{\mathcal{D}_{U^{\dagger} Q}}\left(\mathcal{O}_{U^{\dagger}, Q}, \mathcal{E}^{\dagger}\right), \operatorname{Ext}_{\mathcal{D}_{U^{\dagger}, Q}}^{1}\left(\mathcal{O}_{U^{\dagger}, Q}, \mathcal{E}^{\dagger}\right)
$$

sont de dimension finie.

Traduisons à présent le théorème de finitude de Christol et Mebkhout dans le langage des $\mathcal{D}$-modules arithmétiques de Berthelot:

Proposition 4.6. Si $\mathcal{E}^{\dagger}$ vérifie la propriété $(N L-N L)$, les espaces de cohomologie de $f_{+}\left(\operatorname{sp}_{+}\left(\mathcal{E}^{\dagger}\right)\right)$ sont de dimension finie sur $K$.

DÉmonstration. 1) On a : $\mathbb{R H o m}_{\mathcal{D}_{U^{\dagger},}}\left(\mathcal{O}_{U^{\dagger}, Q}, \mathcal{E}^{\dagger}\right) \stackrel{\sim}{\longrightarrow} \mathbb{R} \Gamma\left(U^{\dagger},-\right) \circ$ $\mathbb{R} \mathcal{H}_{\mathcal{D}_{U^{\dagger}}}\left(\mathcal{O}_{U^{\dagger}, Q}, \mathcal{E}^{\dagger}\right)$. D'après le théorème de type $B$ pour les $\mathcal{O}_{U^{\dagger} Q^{-}}$ modules cohérents (cela se déduit de 1.4), les termes du complexe $\mathbb{R H o m}_{\mathcal{D}_{U^{\dagger},}}\left(\mathcal{O}_{U^{\dagger}, Q}, \mathcal{E}^{\dagger}\right) \stackrel{\sim}{\longrightarrow} \Omega_{U^{\dagger}, \mathrm{Q}}^{\bullet} \otimes_{\mathcal{O}_{U^{\dagger}, Q}} \mathcal{E}^{\dagger}$ sont acycliques pour le foncteur $\Gamma\left(U^{\dagger},-\right)$. On a de plus $\operatorname{RHom}_{D_{U^{\dagger} \mathrm{Q}}}\left(A_{\mathrm{Q}}^{\dagger}, E^{\dagger}\right) \stackrel{\sim}{\longrightarrow} \Omega_{A^{\dagger}, \mathrm{Q}}^{\bullet} \otimes_{A_{Q}^{\dagger}} E^{\dagger}$. Il en résulte :

$$
\mathbb{R H o m}_{\mathcal{D}_{U^{\dagger}}}\left(\mathcal{O}_{U^{\dagger}, Q}, \mathcal{E}^{\dagger}\right) \stackrel{\sim}{\longrightarrow} \mathbb{R} \operatorname{Hom}_{D_{U^{\dagger} Q}}\left(A_{Q}^{\dagger}, E^{\dagger}\right) .
$$


1') De la même façon, grâce aussi à 2.5.1, on obtient le premier isomorphisme :

$$
\begin{aligned}
\operatorname{RHom}_{D_{U^{\dagger}}}\left(A_{\mathrm{Q}}^{\dagger}, E^{\dagger}\right) \stackrel{\sim}{\longrightarrow} \operatorname{RHom}_{\mathcal{D}_{\mathfrak{X}}\left(\dagger Z_{0}\right)_{\mathrm{Q}}}\left(\mathcal{O}_{\mathfrak{X}}\left({ }^{\dagger} Z_{0}\right)_{\mathrm{Q}}, \operatorname{sp}_{+}\left(\mathcal{E}^{\dagger}\right)\right) \stackrel{\sim}{\longrightarrow} \\
\stackrel{\sim}{\longrightarrow} \operatorname{RHom}_{\mathcal{D}_{\mathfrak{X}, Q}}\left(\mathcal{O}_{\mathfrak{X}, \mathrm{Q}}, \operatorname{sp}_{+}\left(\mathcal{E}^{\dagger}\right)\right),
\end{aligned}
$$

le dernier résultant de l'isomorphisme canonique $\mathcal{D}_{\mathfrak{X}}\left({ }^{\dagger} Z_{0}\right)_{\mathrm{Q}} \otimes_{\mathcal{D}_{\mathfrak{X}, Q}} \mathcal{O}_{\mathfrak{X}, \mathrm{Q}} \stackrel{\sim}{\longrightarrow}$ $\mathcal{O}_{\mathfrak{X}}\left({ }^{\dagger} Z_{0}\right)_{\mathrm{Q}}$.

2) Comme l'extension $\mathcal{D}_{\mathfrak{X}, \mathrm{Q}} \rightarrow \mathcal{D}_{\mathfrak{X}, \mathrm{Q}}^{\dagger}$ est plate, comme le morphisme canonique $\mathcal{D}_{\mathfrak{X}, \mathrm{Q}}^{\dagger} \otimes_{\mathcal{D}_{\mathfrak{X}, Q}} \mathcal{O}_{\mathfrak{X}, \mathrm{Q}} \rightarrow \mathcal{O}_{\mathfrak{X}, \mathrm{Q}}$ est un isomorphisme, on obtient alors le premier isomorphisme :

$\operatorname{RHom}_{\mathcal{D}_{\mathfrak{X}, Q}}\left(\mathcal{O}_{\mathfrak{X}, \mathrm{Q}}, \mathrm{sp}_{+}\left(\mathcal{E}^{\dagger}\right)\right) \stackrel{\sim}{\longrightarrow} \operatorname{RHom}_{\mathcal{D}_{\mathfrak{X}, \mathrm{Q}}^{\dagger}}\left(\mathcal{O}_{\mathfrak{X}, \mathrm{Q}}, \mathrm{sp}_{+}\left(\mathcal{E}^{\dagger}\right)\right) \stackrel{\sim}{\longrightarrow} f_{+}\left(\operatorname{sp}_{+}\left(\mathcal{E}^{\dagger}\right)\right)\left[-d_{X}\right]$.

3) En composant les isomorphismes de 1), 1') et 2), on obtient :

$$
\operatorname{RHom}_{\mathcal{D}_{U^{\dagger} Q}}\left(\mathcal{O}_{U^{\dagger}, Q}, \mathcal{E}^{\dagger}\right) \stackrel{\sim}{\longrightarrow} f_{+}\left(\mathrm{sp}_{+}\left(\mathcal{E}^{\dagger}\right)\right)\left[-d_{X}\right] .
$$

On en déduit la proposition grâce au théorème de finitude 4.5.2 de Christol et Mebkhout.

Exemple 4.7. - Si $\mathcal{E}^{\dagger}$ est muni d'une structure de Frobenius, les espaces de cohomologie de $f_{+}\left(\mathrm{sp}_{+}\left(\mathcal{E}^{\dagger}\right)\right)$ sont de dimension finie.

- Considérons l'exemple donné par Berthelot à la fin de [Ber96b] : On prend $\mathcal{V}=Z_{p}, \mathfrak{X}=\widehat{\mathbb{P}}_{\mathcal{V}}^{1}, U_{0}=G_{m k}$. Soit $\alpha \in Z_{(p)}$ un nombre de Liouville, i.e. qui ne vérifie pas la condition $(N L)$. On considère alors l'isocristal surconvergent $\mathcal{E}$ sur $U_{0}$ défini par $\mathcal{O}_{\mathfrak{X}}\left({ }^{\dagger} Z_{0}\right)_{\mathrm{Q}}$ qui est muni de la connection $\nabla$ telle que $\nabla(1)=(\alpha / t) d t$. On calcule alors que le premier espace de cohomologie de $\mathcal{E}$ (le conoyau de $\nabla$ ) n'est pas de dimension finie.

\section{Condition $(N L-N L)$ et stabilité de l'holonomie}

Nous supposons dans ce chapitre que $X_{0}$ est de dimension pure égale à 1 .

Proposition 5.1. Soit $\mathcal{G} \in D_{\mathrm{coh}}^{\mathrm{b}}\left(\mathcal{D}_{\mathfrak{X}, \mathrm{Q}}^{\dagger}\right)\left(\right.$ resp. $\left.\mathcal{G} \in D_{\mathrm{hol}}^{\mathrm{b}}\left(\mathcal{D}_{\mathfrak{X}, \mathrm{Q}}^{\dagger}\right)\right)$ tel que les espaces de cohomologie de $\left.f_{+}\left(\mathcal{G}^{\dagger} Z_{0}\right)\right)$ soient de dimension finie. Alors $\left.\mathcal{G}\left({ }^{\dagger} Z_{0}\right) \in D_{\text {coh }}^{\mathrm{b}}\left(\mathcal{D}_{\mathfrak{X}, \mathrm{Q}}^{\dagger}\right)\left(\operatorname{resp} \cdot \mathcal{G}^{\dagger} Z_{0}\right) \in D_{\text {hol }}^{\mathrm{b}}\left(\mathcal{D}_{\mathfrak{X}, \mathrm{Q}}^{\dagger}\right)\right)$.

DÉMONSTRATION. La preuve est identique à celle de [Car06b, 2.3.2] : si deux des complexes d'un triangle distingué sont cohérents (resp. holo- 
nomes) alors le troisième l'est aussi. En appliquant le foncteur $f_{+}$au triangle de localisation de $\mathcal{G}$ relative à $Z_{0}$, il en résulte la cohérence (resp. holonomie) de $f_{+} \mathbb{R} \underline{\Gamma}_{Z_{0}}^{\dagger}(\mathcal{G})$. Or, via le théorème de Berthelot-Kashiwara, cette cohérence (resp. holonomie) est équivalente à celle de $\mathbb{R} \underline{\Gamma}_{Z_{0}}^{\dagger}(\mathcal{G})$. Grâce au triangle de localisation de $\mathcal{G}$ relative à $Z_{0}$, il en résulte alors celle de $\mathcal{G}$. $\square$

Proposition 5.2. Soit $\mathcal{V} \rightarrow \mathcal{V}^{\prime}$ une extension finie d'anneaux de valuation discrètes complets d'inégales caractéristiques $(0, p)$. On pose $\mathfrak{X}^{\prime} \times_{\operatorname{Spf}(\mathcal{V})} \operatorname{Spf}\left(\mathcal{V}^{\prime}\right)$ le $\mathcal{V}^{\prime}$-schéma formel lisse déduit par changement de base et $\alpha: \mathfrak{X}^{\prime} \rightarrow \mathfrak{X}$ la projection canonique. Soit $\mathcal{G}$ un $\mathcal{D}_{\mathfrak{X}}^{\dagger}\left({ }^{\dagger} Z_{0}\right)_{\mathrm{Q}^{-}-\text {module cohér- }}$ ent. Alors $\mathcal{G}$ est $\mathcal{D}_{\mathfrak{X}, \mathrm{Q}}^{\dagger}$-cohérent (resp. holonome) si et seulement si $\alpha^{*}(\mathcal{G})$ est $\mathcal{D}_{\mathfrak{X}^{\prime}, Q^{\prime}}^{\dagger}$-cohérent (resp. holonome).

Démonstration. Si $\mathcal{G}$ est $\mathcal{D}_{\mathfrak{X}, \mathrm{Q}}^{\dagger}$-cohérent (resp. holonome), il est alors immédiat (voir [Ber02]) que $\alpha^{*}(\mathcal{G})$ soit $\mathcal{D}_{\mathfrak{X}^{\prime}, \mathrm{Q}}^{\dagger}$-cohérent (resp. holonome). Réciproquement, supposons que $\alpha^{*}(\mathcal{G})$ soit $\mathcal{D}_{\mathfrak{X}^{\prime}, Q}^{\dagger}$-cohérent. Comme l'assertion est locale, on se ramène au cas où $\mathfrak{X}$ est affine. Posons $G:=\Gamma(\mathfrak{X}, \mathcal{G})$ et $Z_{0}^{\prime}:=\alpha^{-1}\left(Z_{0}\right)$.

0) Comme dans le cas des $\mathcal{D}$-modules en caractéristique nulle, on vérifie que le morphisme canonique $\mathcal{D}_{\mathcal{X}^{\prime}}^{\dagger}\left({ }^{\dagger} Z_{0}^{\prime}\right)_{\mathrm{Q}} \rightarrow \alpha^{*} \mathcal{D}_{\mathfrak{X}}^{\dagger}\left({ }^{\dagger} Z_{0}\right)_{\mathrm{Q}}$ est en fait un isomorphisme et que le morphisme que l'on en déduit $\alpha^{-1} \mathcal{D}_{\mathcal{X}}^{\dagger}\left({ }^{\dagger} Z_{0}\right)_{\mathbb{Q}} \rightarrow$ $\left.\mathcal{D}_{\mathcal{X}^{\prime}}^{\dagger}{ }^{\dagger} Z_{0}^{\prime}\right)_{\mathrm{Q}}$ est un morphisme d'anneaux (voir [Ber02, 2.2.2]). On obtient ainsi les extensions $D_{\mathfrak{X}}^{\dagger}\left({ }^{\dagger} Z_{0}\right)_{\mathrm{Q}} \rightarrow D_{\mathfrak{X}^{\prime}}^{\dagger}\left({ }^{\dagger} Z_{0}^{\prime}\right)_{\mathrm{Q}}$ et $D_{\mathfrak{X}, \mathrm{Q}}^{\dagger} \rightarrow D_{\mathfrak{X}^{\prime}, \mathrm{Q}}^{\dagger}$.

I.1) Comme $\mathcal{G}$ est $\mathcal{D}_{\mathfrak{X}}^{\dagger}\left({ }^{\dagger} Z_{0}\right)_{\mathrm{Q}}$-cohérent, par théorème de type $A$ : $\Gamma\left(\mathfrak{X}^{\prime}, \alpha^{*}(\mathcal{G})\right) \stackrel{\sim}{\longrightarrow} D_{\mathfrak{X}^{\prime}}^{\dagger}\left({ }^{\dagger} Z_{0}^{\prime}\right)_{\mathrm{Q}} \otimes_{\left.D_{\mathfrak{*}}^{\dagger} \dagger Z_{0}\right)_{Q}} G$. Comme le morphisme canonique $D_{\mathfrak{X}^{\prime}, \mathrm{Q}}^{\dagger} \otimes_{D_{\mathfrak{X}, \mathrm{Q}}^{\dagger}} D_{\mathfrak{X}}^{\dagger}\left({ }^{\dagger} Z_{0}\right)_{\mathrm{Q}} \rightarrow D_{\mathfrak{X}^{\prime}}^{\dagger}\left({ }^{\dagger} Z_{0}^{\prime}\right)_{\mathrm{Q}}$ est un isomorphisme, il en résulte $\Gamma\left(\mathfrak{X}^{\prime}, \alpha^{*}(\mathcal{G})\right) \stackrel{\sim}{\longrightarrow} D_{\mathfrak{X}^{\prime}, Q}^{\dagger} \otimes_{D_{\mathfrak{x}, Q}^{\dagger}} G$.

I.2) Or, d'après un théorème de type $A, \Gamma\left(\mathfrak{X}^{\prime}, \alpha^{*}(\mathcal{G})\right)$ est $D_{\mathfrak{X}^{\prime}, Q^{\dagger}}^{\dagger}$-cohérent. De plus, le morphisme $D_{\mathfrak{X}, \mathrm{Q}}^{\dagger} \rightarrow D_{\mathfrak{X}^{\prime}, \mathrm{Q}}^{\dagger}$ est fidèlement plat car il se déduit par extension du morphisme $\mathcal{V} \rightarrow \mathcal{V}^{\prime}$. Cela implique alors que $G$ est un $D_{\mathfrak{X}, Q^{-}}^{\dagger}$ module cohérent.

II) Soit $a$ une section globale de $\mathcal{O}_{\mathfrak{X}}$ et notons $a^{\prime}$ la section globale de $\mathcal{O}_{\mathfrak{X}^{\prime}}$ déduite. De manière analogue à I.1), comme $\mathcal{G} \mid \mathfrak{X}_{a}$ est $\mathcal{D}_{\mathfrak{X}_{a}}^{\dagger}\left({ }^{\dagger} Z_{0} \cap X_{a}\right)_{Q^{-}}$-cohérent, on vérifie

$$
\Gamma\left(\mathfrak{X}_{a^{\prime}}^{\prime}, \alpha^{*}(\mathcal{G})\right) \stackrel{\sim}{\longrightarrow} D_{\mathfrak{X}_{a^{\prime}}^{\prime}, \mathrm{Q}}^{\dagger} \otimes_{D_{\mathfrak{x}_{a}, Q}^{\dagger}} \Gamma\left(\mathfrak{X}_{a}, \mathcal{G}\right) .
$$

Comme $\alpha^{*}(\mathcal{G})$ est $\mathcal{D}_{\mathfrak{X}^{\prime}, Q^{\prime}}^{\dagger}$-cohérent, $\Gamma\left(\mathfrak{X}_{a^{\prime}}^{\prime}, \alpha^{*}(\mathcal{G})\right)$ est $D_{\mathfrak{X}_{a^{\prime}}^{\prime}, Q^{\dagger}}^{\dagger}$-cohérent et 
$\Gamma\left(\mathfrak{X}_{a^{\prime}}^{\prime}, \alpha^{*}(\mathcal{G})\right) \stackrel{\sim}{\longrightarrow} D_{\mathfrak{X}_{a^{\prime}, Q}^{\prime}}^{\dagger} \otimes_{D_{\mathfrak{X}^{\prime}, Q}^{\dagger}} \Gamma\left(\mathfrak{X}^{\prime}, \alpha^{*}(\mathcal{G})\right)$. Via la conclusion de I.1), il en découle le premier isomorphisme :

$$
\Gamma\left(\mathfrak{X}_{a^{\prime}}^{\prime}, \alpha^{*}(\mathcal{G})\right) \stackrel{\sim}{\longrightarrow} D_{\mathfrak{X}_{a^{\prime}}^{\prime}, \mathrm{Q}}^{\dagger} \otimes_{D_{\mathfrak{*}, Q}^{\dagger}} G \stackrel{\sim}{\longrightarrow} D_{\mathfrak{X}_{a^{\prime}}^{\prime}, \mathrm{Q}}^{\dagger} \otimes_{D_{\mathfrak{x} a, Q}^{\dagger}}\left(D_{\mathfrak{X}_{a}, Q}^{\dagger} \otimes_{D_{\mathfrak{x}, Q}^{\dagger}}^{\dagger} G\right) .
$$

Comme l'extension $D_{\mathfrak{X}_{a}, \mathrm{Q}}^{\dagger} \rightarrow D_{\mathfrak{X}_{a^{\prime}}, \mathrm{Q}}^{\dagger}$ est fidèlement plate, il en résulte que le morphisme canonique $D_{\mathfrak{x}_{a}, \mathrm{Q}}^{\dagger} \otimes_{D_{\mathfrak{x},}^{\dagger}}^{\dagger} G \rightarrow \Gamma\left(\mathfrak{X}_{a}, \mathcal{G}\right)$ est un isomorphisme. D'après le théorème de type $A$ pour les $\mathcal{D}_{\mathfrak{X}, \mathrm{Q}}^{\dagger}$-modules cohérents, cela implique la $\mathcal{D}_{\mathfrak{X}, \mathrm{Q}}^{\dagger}$-cohérence de $\mathcal{G}$.

III) $\mathrm{Si} \alpha^{*}(\mathcal{G})$ est un $\mathcal{D}_{\mathfrak{X}^{\prime}, Q^{\dagger}}^{\dagger}$-module holonome alors d'après l'étape précédente, $\mathcal{G}$ est un $\mathcal{D}_{\mathfrak{X}, \mathrm{Q}}^{\dagger}$-module cohérent. Comme l'extension $\alpha^{-1} \mathcal{D}_{\mathfrak{X}, \mathrm{Q}}^{\dagger} \rightarrow$ $\mathcal{D}_{\mathfrak{X}^{\prime}, \mathrm{Q}}^{\dagger}$ est plate, comme $\alpha^{*}(\mathcal{G})$ est holonome, il en résulte que $\mathcal{G}$ est holonome.

Remarque 5.3. Grâce à la proposition 5.2, nous pourrons utiliser le théorème d'algébrisation de Christol et Mebkhout de 4.5 dont le résultat n'est garanti qu'après une extension finie de la base.

DÉFInition 5.4 Soient $T_{0}$ un diviseur $\operatorname{de} X_{0}$ et $\mathcal{G} \in D_{\text {coh }}^{\mathrm{b}}\left(\mathcal{D}_{\mathfrak{X}}^{\dagger}\left({ }^{\dagger} T_{0}\right)_{\mathrm{Q}}\right)$. On dit que $\mathcal{G}$ vérifie la propriété $(N L-N L)$ s'il existe un diviseur $Z^{\prime}$ de $X$ tel que $U^{\prime}:=X \backslash Z^{\prime}$ soit affine, $Z_{0}^{\prime}:=Z^{\prime} \otimes_{\mathcal{V}} k \supset T_{0}$ et, pour tout $\left.l \in Z, \mathcal{H}^{l} \mathcal{G}^{(}{ }^{\dagger} Z_{0}^{\prime}\right)$ soit associé à un isocristal surconvergent sur $U_{0}^{\prime}:=U^{\prime} \otimes_{\mathcal{V}} k$ qui vérifie la propriété $(N L-N L)$.

EXemple 5.5. Avec les notations de 5.4, en notant $\mathcal{Y}:=\mathfrak{X} \backslash T_{0}$, les $F$ complexes $\mathcal{G}$ de $F-D_{\text {coh }}^{\mathrm{b}}\left(\mathcal{D}_{\mathfrak{X}}^{\dagger}\left({ }^{\dagger} T_{0}\right)_{\mathrm{Q}}\right)$ tels que $\mathcal{G} \mid \mathcal{Y}$ est holonome vérifient la propriété $(N L-N L)$. En effet, cela résulte de [Ber02, 5.3.5.(i)], [Car06b, 2.2.12] et du fait qu'un $F$-isocristal surconvergent sur une courbe lisse vérifie la propriété $(N L-N L)$. Le théorème ci-dessous est donc une extension au cas sans structure de Frobenius de la conjecture «forte» de Berthelot (voir [Ber02, 5.3.6.(D)]).

THÉORÈme 5.6 Soient $T_{0}$ un diviseur de $X$ et $\mathcal{G} \in D_{\text {coh }}^{\mathrm{b}}\left(\mathcal{D}_{\mathfrak{X}}^{\dagger}\left({ }^{\dagger} T_{0}\right)_{\mathbb{Q}}\right)$ satisfaisant à la propriété $(N L-N L)$. Alors $\mathcal{G} \in D_{\text {hol }}^{\mathrm{b}}\left(\mathcal{D}_{\mathfrak{X}, \mathrm{Q}}^{\dagger}\right)$.

DÉmonstration. Grâce à 5.2 et au théorème d'algébrisation de Christol et Mebkhout de 4.5, quitte à faire une extension finie de la base, on supposer qu'il existe un diviseur $Z^{\prime}$ de $X$ tel que $U^{\prime}:=X \backslash Z^{\prime}$ soit affine, 
$Z_{0}^{\prime}:=Z^{\prime} \otimes_{\mathcal{V}} k \supset T_{0}$ et, pour tout $\left.l \in Z, \mathcal{H}^{l} \mathcal{G}^{\dagger} Z_{0}^{\prime}\right)$ soit un isocristal surconvergent sur $U^{\prime} \otimes_{\mathcal{V}} k$ qui vérifie la propriété $(N L-N L)$ et qui soit dans l'image essentielle du foncteur daguification de 3.1.1. D'après 3.9 et 3.10.1, il existe alors un $\mathcal{D}_{\mathfrak{X}, \mathrm{Q}}^{\dagger}$-module holonome $\mathcal{G}_{l}$ tel que $\mathcal{H}^{l} \mathcal{G}\left({ }^{\dagger} Z_{0}^{\prime}\right) \stackrel{\sim}{\longrightarrow} \mathcal{G}_{l}\left({ }^{\dagger} Z_{0}^{\prime}\right)$. De plus, il découle de la traduction 4.6 du théorème de finitude de Christol et Mebkhout que les espaces de cohomologie de $f_{+}\left(\mathcal{H}^{l} \mathcal{G}\left({ }^{\dagger} Z_{0}^{\prime}\right)\right)$ sont de dimension finie sur $K$. Il résulte alors de 5.1 que $\mathcal{H}^{l} \mathcal{G}\left({ }^{\dagger} Z_{0}^{\prime}\right)$ est un $\mathcal{D}_{\mathfrak{X}, Q^{-}}^{\dagger}$ module holonome. On a ainsi établi que $\left.\mathcal{G}^{\dagger} Z_{0}^{\prime}\right) \in D_{\text {hol }}^{\mathrm{b}}\left(\mathcal{D}_{\mathfrak{X}, \mathrm{Q}}^{\dagger}\right)$. On peut supposer $Z_{0}^{\prime}$ et $T_{0}$ réduits. Soit $Z_{0}^{\prime \prime}$ tel que $Z_{0}^{\prime}$ soit la réunion disjointe de $T_{0}$ et $Z_{0}^{\prime \prime}$. Comme $\mathcal{G}\left({ }^{\dagger} Z_{0}^{\prime}\right) \stackrel{\sim}{\longrightarrow} \mathcal{G}\left({ }^{\dagger} Z_{0}^{\prime \prime}\right)$ (e.g. cela découle de [Car04, 2.2.14] et du fait que $\left.\left.\mathcal{G} \in D_{\text {coh }}^{\mathrm{b}}\left(\mathcal{D}_{\mathfrak{X}}^{\dagger}{ }^{\dagger} T_{0}\right)_{\mathrm{Q}}\right)\right)$, on déduit du triangle de localisation de $\mathcal{G}$ en $Z_{0}^{\prime \prime}$ que $\mathbb{R}{\underline{\Gamma^{\prime \prime}}}_{Z_{0}^{\prime \prime}}^{\dagger}(\mathcal{G}) \in D_{\text {coh }}^{\mathrm{b}}\left(\mathcal{D}_{\mathfrak{X}}^{\dagger}\left({ }^{\dagger} T_{0}\right)_{\mathbb{Q}}\right)$. Soient $u: \mathcal{Z}^{\prime \prime} \hookrightarrow \mathfrak{X}$ un relèvement de $Z_{0}^{\prime \prime} \rightarrow \mathfrak{X}$. Comme $Z_{0}^{\prime \prime} \cap T_{0}$ est vide, comme $u_{+} u^{\prime}(\mathcal{G}) \stackrel{\sim}{\longrightarrow} \mathbb{R} \underline{\Gamma}_{Z_{0}^{\prime \prime}}^{\dagger}(\mathcal{G})$ est à support dans $Z_{0}^{\prime \prime}$, il résulte alors du théorème de Berthelot-Kashiwara que $u^{!}(\mathcal{G})$ est un isocristal convergent sur $Z_{0}^{\prime \prime}$ (comme $Z_{0}^{\prime \prime}$ est de dimension nulle, être un $\mathcal{D}_{0}^{\dagger}$-module cohérent sur $Z_{0}^{\prime \prime}$ ou un isocristal convergent sur $Z_{0}^{\prime \prime}$ sont deux choses équivalentes). En particulier, le module $u^{!}(\mathcal{G})$ est holonome (les isocristaux convergents sont toujours holonomes car leur dual comme $\mathcal{D}_{Q}^{\dagger}$-module est le même que comme isocristal convergent, ce dernier n'ayant qu'un terme : voir [Car05]). Comme $u$ est une immersion fermée, le foncteur $u_{+}$préserve l'holonomie (en effet, cela résulte du théorème de dualité relative et de l'exactitude de $u_{+}$). On en déduit que $\mathbb{R} \underline{\Gamma}_{Z_{0}^{\prime \prime}}^{\dagger}(\mathcal{G}) \in D_{\text {hol }}^{\mathrm{b}}\left(\mathcal{D}_{\mathfrak{X}, \mathrm{Q}}^{\dagger}\right)$. Via le triangle de localisation de $\mathcal{G}$ en $Z_{0}^{\prime \prime}$, il en dérive que $\mathcal{G} \in D_{\text {hol }}^{\mathrm{b}}\left(\mathcal{D}_{\mathfrak{X}, \mathrm{Q}}^{\dagger}\right)$.

Corollaire 5.7. Soient $T_{0}$ un diviseur de $X$ et $\mathcal{G}$ un $\mathcal{D}_{\mathcal{X}}^{\dagger}\left({ }^{\dagger} T_{0}\right)_{\mathrm{Q}}$-module cohérent, $\mathcal{O}_{\mathfrak{X}}\left({ }^{\dagger} T_{0}\right)_{\mathrm{Q}}$-cohérent qui vérifie la propriété $(N L-N L)$. Alors $\mathcal{G}$ est un $\mathcal{D}_{\mathfrak{X}, \mathrm{Q}}^{\dagger}$-module holonome.

\section{REFERENCES}

[Ber90] P. Berthelot, Cohomologie rigide et théorie des $\mathcal{D}$-modules, $p$-adic analysis (Trento, 1989) (Springer, Berlin, 1990), pp. 80-124.

[Ber96a] P. BERTHELOT, Cohomologie rigide et cohomologie rigide à support propre. Première partie, Prépublication IRMAR 96-03, Université de Rennes, 1996.

[Ber96b] P. BERThelot, [-modules arithmétiques. I. Opérateurs différentiels de niveau fini, Ann. Sci. École Norm. Sup. (4) 29, no. 2 (1996), pp. 185-272.

[Ber02] P. BERTHELOT, Introduction à la théorie arithmétique des D-modules, Astérisque, no. 279 (2002), pp. 1-80, Cohomologies p-adiques et applications arithmétiques, II. 
[Car04] D. CARo, D-modules arithmétiques surcohérents. Application aux fonctions L, Ann. Inst. Fourier, Grenoble 54, no. 6 (2004), pp. 1943-1996.

[Car05] D. CARo, Comparaison des foncteurs duaux des isocristaux surconvergents, Rend. Sem. Mat. Univ. Padova 114 (2005), pp. 131-211.

[Car06a] D. CARo, Dévissages des F-complexes de D-modules arithmétiques en F-isocristaux surconvergents, Invent. Math. 166, no. 2 (2006), pp. 397456.

[Car06b] D. CARo, Fonctions Lassociées aux D-modules arithmétiques. Cas des courbes, Compositio Mathematica 142, no. 01 (2006), pp. 169-206.

[Car07] D. CARo, Overconvergent F-isocrystals and differential overcoherence, Invent. Math. 170, no. 3 (2007), pp. 507-539.

[Car09] D. CARo, D-modules arithmétiques surholonomes, Ann. Sci. École Norm. Sup. (4) 42, no. 1 (2009), pp. 141-192.

[Car11a] D. CARo, Holonomie sans structure de frobenius et critères d'holonomie, À paraître aux Ann. Inst. Fourier (Grenoble) (2011).

[Car11b] D. CARo, Pleine fidélité sans structure de Frobenius et isocristaux partiellement surconvergents, Math. Annalen 349 (2011), pp. 747-805.

[Car11c] D. CARo, Stability of holonomicity over quasi-projective varieties, À paraître dans Compositio Mathematica (2011).

[CM97] G. Christol - Z. Meвкhout, Sur le théorème de l'indice des équations différentielles p-adiques. II, Ann. of Math. (2) 146, no. 2 (1997), pp. 345410.

[CM00] G. Christol - Z. Mebkhout, Sur le théorème de l'indice des équations différentielles p-adiques. III, Ann. of Math. (2) 151, no. 2 (2000), pp. 385457.

[CM01] G. Christol - Z. Mebkhout, Sur le théorème de l'indice des équations différentielles p-adiques. IV, Invent. Math. 143, no. 3 (2001), pp. 629672.

[CT08] D. CARO - N. TsuzUKI, Overholonomicity of overconvergent F-isocrystals over smooth varieties, ArXiv Mathematics e-prints (2008).

[Mer72] D. Meredith, Weakformal schemes, Nagoya Math. J.45 (1972), pp. 1-38.

MW68] P. Monsky - G. WAshnitzer, Formal cohomology. I, Ann. of Math. (2) 88 (1968), pp. 181-217.

[NH03] C. NoOT-HuYghe, Un théorème de comparaison entre les faisceaux d'opérateurs différentiels de Berthelot et de Mebkhout-Narváez-Macarro, J. Algebraic Geom. 12, no. 1 (2003), pp. 147-199.

[Vir00] A. VIRRION, Dualité locale et holonomie pour les $\mathcal{D}$-modules arithmétiques, Bull. Soc. Math. France 128, no. 1 (2000), pp. 1-68.

Manoscritto pervenuto in redazione il 24 novembre 2010. 